\title{
Medium-energy particle experiments- electron analyzer (MEP-e) for the exploration of energization and radiation in geospace (ERG) mission
}

\author{
Satoshi Kasahara ${ }^{1 *} \mathbb{D}$, Shoichiro Yokota ${ }^{2}$, Takefumi Mitani ${ }^{3}$, Kazushi Asamura $^{3}$, Masafumi Hirahara ${ }^{4}$, \\ Yasuko Shibano ${ }^{3}$ and Takeshi Takashima ${ }^{3}$
}

\begin{abstract}
The medium-energy particle experiments - electron analyzer onboard the exploration of energization and radiation in geospace spacecraft measures the energy and direction of each incoming electron in the energy range of 7-87 keV. The sensor covers a $2 \pi$-radian disklike field of view with 16 detectors, and the full solid angle coverage is achieved through the spacecraft's spin motion. The electron energy is independently measured by both an electrostatic analyzer and avalanche photodiodes, enabling significant background reduction. We describe the technical approach, data output, and examples of initial observations.
\end{abstract}

Keywords: Radiation belts, ERG spacecraft, Medium-energy particles

\section{Introduction}

The exploration of energization and radiation in geospace (ERG) project is designed to explore the Earth's radiation belt region, where relativistic-energy electrons, with energy of the order of $\mathrm{MeV}$, are generated from considerably lower-energy source populations, such as solar wind electrons with energy of hundreds of $\mathrm{eV}$ and electrons from ionospheric sources with sub-eV energy (Miyoshi et al. 2017). The ERG spacecraft was launched from the Uchinoura space center in Kagoshima, Japan, at 11:00 UTC on December 20, 2016, and thereafter was nicknamed "Arase," after a wild river near the launch site. The spacecraft altitude is $440 \mathrm{~km}$ in perigee and 32,000 km in apogee after the initial maneuvering, with an inclination of $\sim 31^{\circ}$. For the extensive plasma measurements, the spacecraft is equipped with eight sensors for particles and fields and one software-type analyzer.

\footnotetext{
*Correspondence: s.kasahara@eps.s.u-tokyo.ac.jp

${ }^{1}$ Department of Earth and Planetary Science, School of Science, The

University of Tokyo, 7-3-1, Hongo, Bunkyo-ku, Tokyo, Japan

Full list of author information is available at the end of the article
}

The medium-energy particle experiments-electron analyzer (MEP-e) is one of these instruments. It measures the energy and direction of each incoming electron in the medium-energy range $(7-87 \mathrm{keV})$. This energy range is key to understanding the formation and decay of the radiation belt, as these electrons excite whistler-mode waves (Kennel and Petchek 1966; Omura and Summers 2006; Omura et al. 2008), which have been theoretically suggested to play significant roles in the acceleration and loss of electrons (Omura and Summers 2006; Summers et al. 1998, Horne et al. 2005; Katoh and Omura 2006, 2007; Hikishima et al. 2010). Furthermore, they are important as the seed population for the relativistic electrons (Horne et al. 2007). These relationships are schematically summarized in Fig. 1.

Using MEP-e, we obtain the velocity distribution functions of medium-energy electrons, providing key information regarding the local energization and pitch-angle scattering, as well as on the global dynamics. The main topics to be addressed with MEP-e are the (1) enhancement and decay of the electron ring current, which is the seed population for higher-energy electrons, (2) evolution of pitch-angle distributions during flux increase/ 


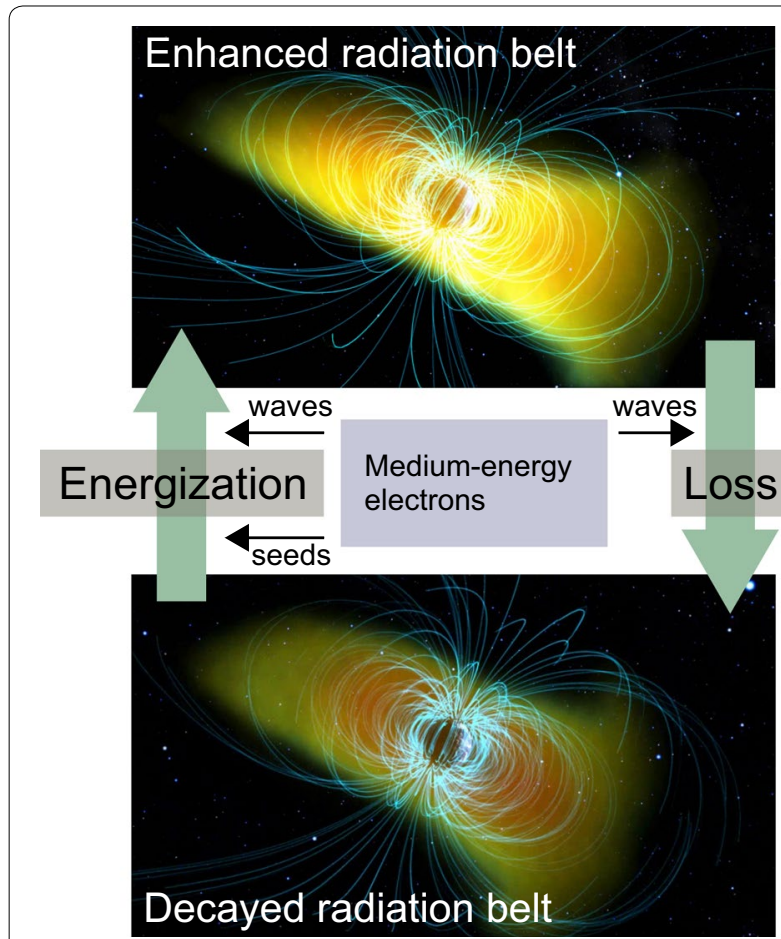

Fig. 1 Schematic showing the enhancement and decay of the radiation belt. The top and bottom images show the enhanced and decayed radiation belts, respectively. For the acceleration and loss of MeV-range electrons, the medium-energy electrons play important roles as seed populations and through wave generations decrease, and (3) energy transfer between electrons and electromagnetic waves via Landau/gyro-resonances. All observations contribute to the determination of the mechanisms of generation and loss of relativistic electrons in the radiation belt. This paper describes the measurement principle of MEP-e, presents the ground calibration results, and illustrates the in-flight performances.

\section{Overview of the instrument}

Figure 2a shows the MEP-e flight model. The upper cylindrical unit, which includes the sensor optics, detectors, and a part of the electronics boards, sticks out from the panel, while the lower box unit consisting of the CPU and two power supply unit (PSU) boards is inside the spacecraft chassis. The sensor aperture for the electrons is the slit over $2 \pi$ radians near the top of the cylindrical structure, which is easily recognized in Fig. 2b, representing the cross-sectional view of the MEP-e. The incoming electrons are filtered by an electrostatic analyzer (ESA) with regard to their incident energies and then detected by avalanche photodiodes (APDs). The velocity distribution functions over the medium-energy range are obtained by sweeping the high voltage applied to the ESA (thus scanning energy) and determining the incoming directions by sensing each signal with discrete detectors. Sixteen APDs are mounted azimuthally. More technical

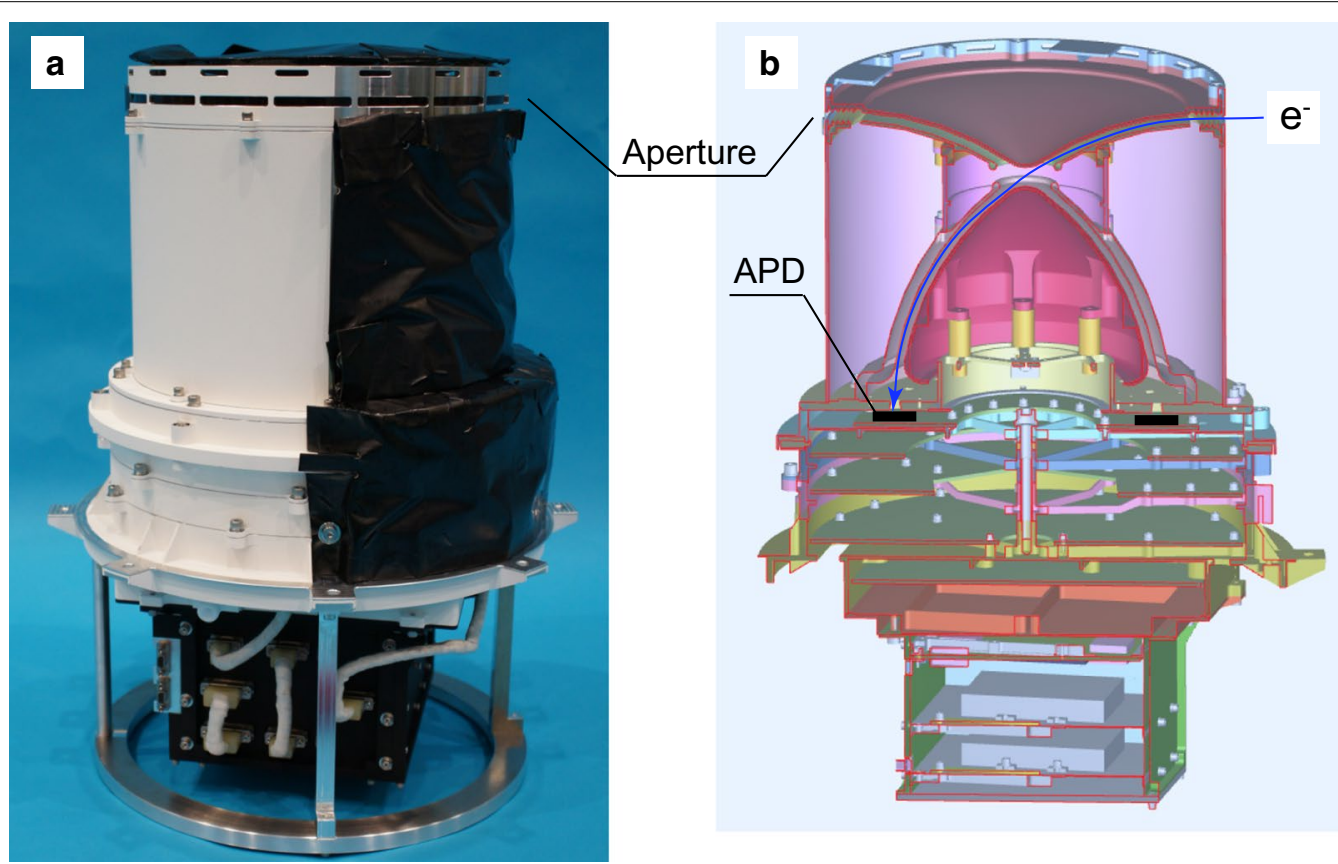

Fig. 2 Sensor structure of the MEP-e. The side facing to the sun [the right in $\mathbf{a}$ ] and the top are covered by black kapton MLIs, while the left side is painted white for cooling. The cross-sectional view and an incoming electron trajectory are shown in $\mathbf{b}$ 
details of the ESA and APDs are described in "Measurement techniques" section.

The location of the MEP-e on the spacecraft is illustrated in Fig. 3a. Although two other particle instruments (HEP, Mitani et al. 2017, and LEP-i, Asamura et al. 2017) are on the same panel of the spacecraft, the field of view (FOV) of the MEP-e is not blocked by these analyzers, since MEP-e is the tallest. However, the FOV of the MEP-e is slightly blocked by the solar array paddle (SAP) yokes and wire antennas of PWE (Kasahara et al. 2017), as shown in Fig. 3b, although the effects are mostly negligible. A noticeable count rate reduction is seen only in azimuth channel 9 , by $\sim 10 \%$ (based on in-flight data) due to the SAP yoke. This is taken into account at the ground calibration.

ERG is a sun-pointing spinning spacecraft, and the measurement of the MEP-e is in principle synchronized with the spacecraft spin. The spin is sectored by 32 (i.e., 32 spin-phase channels, $11.25^{\circ}$ each), and the applied high voltage is swept through 16 steps in each spin phase for the energy scan. The time cadence for the data acquisition is adjusted at each spin, based on the previous spin period. The sequence is shown in Fig. 3c. Because the nominal spin period of the ERG is $\sim 8 \mathrm{~s}$, each SV (sweeping voltage) step is $\sim 15.6 \mathrm{~ms}$ ( $=8 \mathrm{~s} / 32$-spin-phase/16-SV-step).

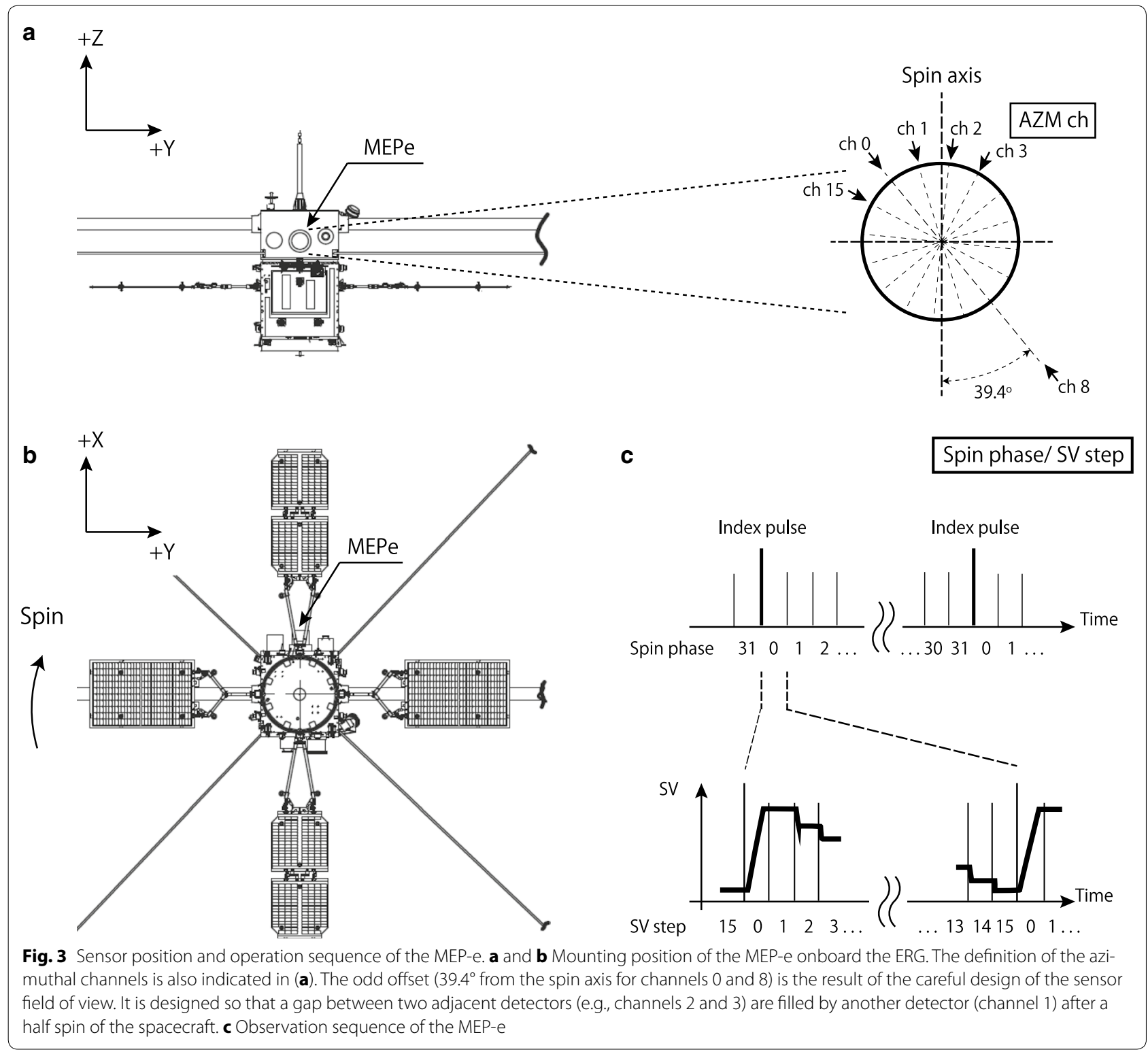




\section{Specifications}

Table 1 summarizes the specifications of the MEP-e. The energy range and resolution, FOV, size, weight, and power consumption are based on the actual measurements for the flight model. The energy steps are controlled by the sensor's field-programmable gate array (FPGA), using lookup tables that contain the SV values for 16 steps. These tables are rewritable by command after the launch. The geometric factor is determined using a computer simulation. The description of the time resolution is based on the nominal spin period of $\sim 8 \mathrm{~s}$. The sensor size, mass, and power consumption include those of the CPU and PSU.

Figure 4 illustrates the function block diagram of the MEP-e. When an electron comes into the sensor and is detected by an APD, the amount of the output charge is converted to the voltage pulse height $(\mathrm{PH})$ by a chargesensitive amplifier (CSA). After the pulse shaping (at a shaper), the signal peak is held at the peak holder and converted into a digital value by the analog-to-digital converter $(\mathrm{ADC})$. The conversion is triggered when the shaped pulse exceeds the discrimination level that is set by the FPGA. When the conversion is performed, a reset pulse is sent to release the peak-held signal. The dead time, corresponding to the duration between the electron incidence and the peak-hold reset, is $\sim 10 \mu \mathrm{s}$. There are 16 amplifier circuits in parallel (each correspond to an APD) and four ADC chips. Thus, each of the ADC chips handles four APD signal lines with a multiplexer. These $\mathrm{PH}$ analyses are controlled by the sensor FPGA.

Due to the resource of the FPGA, the parallel (simultaneous) signal handling is limited to two lines per ADC chip. This implies that, for a single ADC chip, the third or later signals within the dead time are ignored. Assuming that saturation becomes gradually significant at a signal rate of $\sim 20 \mathrm{kHz}$ per ADC chip (four signal lines), for which the average interval is five times the dead time, the corresponding count rate per azimuth channel and the energy differential flux are $5 \mathrm{kHz}$ and $\sim 10^{8} \mathrm{keV} /$ $\mathrm{cm}^{2} \mathrm{sr} \mathrm{keV}$ s, respectively. Above this flux level, the saturation (dead time) may occur.

The sensor FPGA also controls and monitors the highvoltage board, which consists of an SV output and four outputs for the APDs (APD-HV, four APDs per each output). The maximum output of the SV is $\sim 5 \mathrm{kV}$ corresponding to the measurement energy of $\sim 87 \mathrm{keV}$. The maximum output of the APD-HV is $-250 \mathrm{~V}$, while the nominal value is around -160 to $170 \mathrm{~V}$. The gain of the APD (ratio of the output PH to the incident energy) depends on the temperature, and therefore, the temperature of the APDs is necessary to determine the incident electron energies. For this purpose, four temperature sensors are mounted on the APDv board. Two other temperature sensors are also installed on the sensor chassis, with two survival heaters shown in Fig. 4 (for the engineering purpose). One is on the side wall of the ESA, below the black kapton MLI shown in Fig. 2a. The other is in the electronics box. These heaters are controlled by the spacecraft bus heater system.

All electronics boards are powered by the PSU1 (digital 3.3 V) and PSU2 $( \pm 12 \mathrm{~V})$. Both of these power supply boards, as well as the CPU board, are common for ERG scientific instruments, except for the differences in the PSU2 output values. The PSU1 and PSU2 are powered by the bus power supply of nominally $\sim 44 \mathrm{~V}$.

The application software in the CPU interfaces with the sensor FPGA and the mission bus network. The sensor FPGA receives the spin pulse and associated time

Table 1 Specification of the MEP-e

\begin{tabular}{|c|c|c|}
\hline Parameter & Value & Notes \\
\hline Energy range & $7-87 \mathrm{keV}$ & \\
\hline Energy resolution & $8 \%$ & FWHM of the electrostatic analyzer response \\
\hline Energy steps & 16 steps per scan & \\
\hline Sensor field of view & $\begin{array}{l}360^{\circ} \text { (azimuth) } \times 3.5^{\circ} \text { (elevation) } \\
\sim 3.5^{\circ} \text { (azimuth) } \times 3.5^{\circ} \text { (elevation) per detector }\end{array}$ & $\begin{array}{l}\text { Azimuthal gaps exist between detectors } \\
\text { FWHM }\end{array}$ \\
\hline Number of APDs & 16 & Circularly aligned \\
\hline Geometric factor & $6.6 \times 10^{-5} \mathrm{~cm}^{2} \mathrm{sr} \mathrm{keV} / \mathrm{keV}$ per detector & APD efficiency not included \\
\hline Time resolution & $\begin{array}{l}4 \mathrm{~s} \text { for the } 3-\mathrm{D} \text { distribution function } \\
250 \mathrm{~ms} \text { for one energy scan } \\
15.6 \mathrm{~ms} \text { for one energy step } \\
\sim 1.5 \mathrm{\mu s} \text { for the S-WPIA data }\end{array}$ & For nominal spacecraft spin (8s) \\
\hline Sensor size & $\varphi 318 \mathrm{~mm} \times 395 \mathrm{~mm}$ & Including PSU/CPU \\
\hline Sensor mass & $8.2 \mathrm{~kg}$ & \\
\hline Power consumption & $21 \mathrm{~W}$ & Including DC/DC converter efficiency \\
\hline Data rate & 1.756 kB per one energy scan & \\
\hline
\end{tabular}




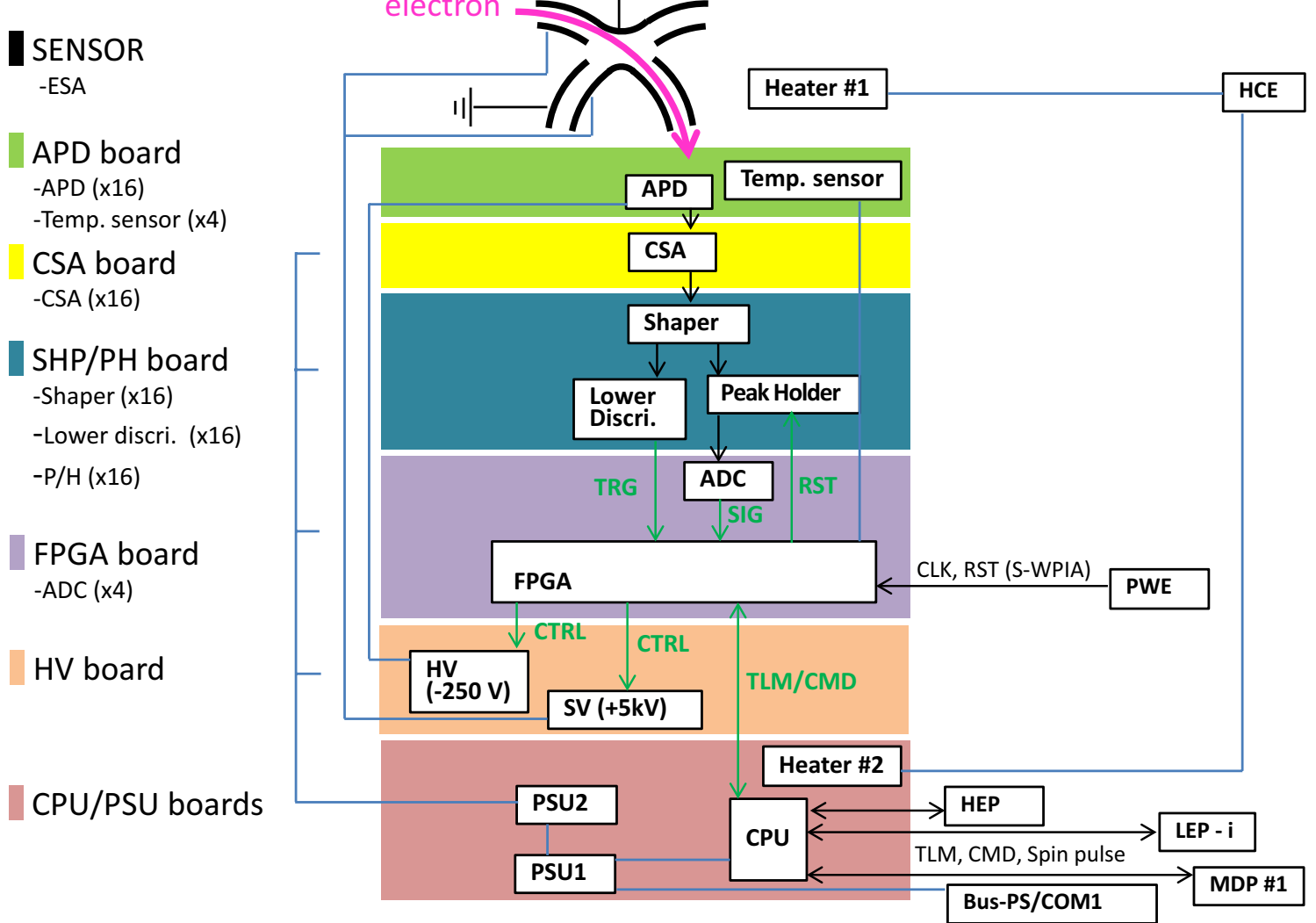

Fig. 4 Block diagram of the MEP-e. The sensor has 16 signal channels in parallel

indicators from a CPU middleware for synchronization, as well as sensor commands, while it sends science data at every spin phase and HK data every $1 \mathrm{~s}$ to the CPU. The software acquires the data via the middleware, edits the obtained data, and sends them to the mission data processor. SpaceWire is used for this communication between the sensor FPGA and CPU and between the CPUs of the mission instruments. The CPU of the MEP-e is nominally connected to those of the HEP and LEP-i and one of the mission data processor/recorders.

\section{Measurement techniques}

Despite their scientific importance, medium-energy electrons occur in an energy range gap between low-energy plasma sensors and high-energy particle detectors. This is due to the low-energy $(<30 \mathrm{keV})$ particles that are conventionally measured by electrostatic analyzers, while the high-energy ( $>50 \mathrm{keV}$ ) particles are covered by solidstate detectors. Both techniques have difficulties regarding accuracy of the measurements at the ends of their energy ranges.

For the measurements of medium-energy electrons onboard the ERG, we designed an electron sensor consist of a cusp-type electrostatic analyzer and APDs. The ESA determines the energy of an incoming electron, while rejecting ions and photons. The APDs are used instead of the classical electron detectors, such as microchannel plates (MCPs) and channel electron multipliers (CEMs), because the quantum efficiencies of MCPs and CEMs fall off at energies above a few $\mathrm{keV}$ and it has been difficult to accurately predict the efficiency curve for the mediumenergy range. Furthermore, the signal charge multiplication by the APDs, enhancing the signal-to-noise ratio, is a significant advantage over classical solid-state detectors. In addition, the ability of the APDs to measure particle (and photon) energies is especially useful for background reduction during observations in a harsh radiation environment, since spurious signals are discarded by a consistency check of the energy, determined by the ESA and APDs independently.

\section{Cusp-type electrostatic analyzer}

For the energy determination by the MEP-e, the cusptype electrostatic analyzer (Kasahara et al. 2006) was utilized. This new type of analyzer was designed specifically for medium-energy particle measurements to reduce 
the size of the sensor compared to conventional ESAs. Figure 5 shows the cross-sectional view of the key components of the cusp-type ESA: upper-outer (larger curvature), upper-inner (smaller-curvature), lower-outer, and lower-inner electrodes, as well as a base plate. The exit holes are seen at the base plate. Compared to the conventional top-hat-type analyzers (Carlson and McFadden 2013), the center of curvature of the plates is far from the sensor axis, resulting in the remarkably small size while keeping the large curvature radius (and thus the high uppermost measurement energy). The curvature radii of the inner and outer electrodes are 150.0 and $154.7 \mathrm{~mm}$, respectively. The maximum electric field strength is thus $5 \mathrm{kV} / 4.7 \mathrm{~mm}-1.06 \mathrm{kV} / \mathrm{mm}$. The size of the exit slit is $4.7 \mathrm{~mm} \times 4.7 \mathrm{~mm}$, which is slightly smaller than the detector window (see below). A high voltage of up to $5 \mathrm{kV}$ is applied to the inner (i.e., smaller-curvature) electrodes. Other electrodes are grounded. The inner surfaces of the outer electrodes are coated by a conductive blackpaint, in order to suppress the EUV solar photons.
APD

As mentioned above, it is difficult to determine the quantum efficiency using the MCPs and CEMs, especially for $\mathrm{keV}$ electrons, without the double-signal coincidence technique (cf., Funsten et al. 2013), and knowledge of the efficiency is essential for appropriately converting the acquired electron count rate (observed value) to the flux (physically meaningful value). Therefore, for the MEPe, we utilized the APD with an efficiency close to unity. The factor which diminishes the APD efficiency from unity is the backscattering of incident electrons at a surface dead layer $(\sim 0.25 \mu \mathrm{m}$ thickness, see Kasahara et al. 2012), since such electrons do not generate significant pulses. The backscattering ratio, $\beta(E)$, represents the ratio of the backscattered electrons to all incident electrons with incident energy $E$ and can be predicted by the wellestablished electron behavior in materials (e.g., Joy 1991). The model efficiency $\varepsilon(E)=1-\beta(E)$ is shown in Fig. 6, ranging from 0.8 to 1.0 for the MEP-e energy range. Although an in-flight precise evaluation of an absolute efficiency is difficult, such a high value and moderate

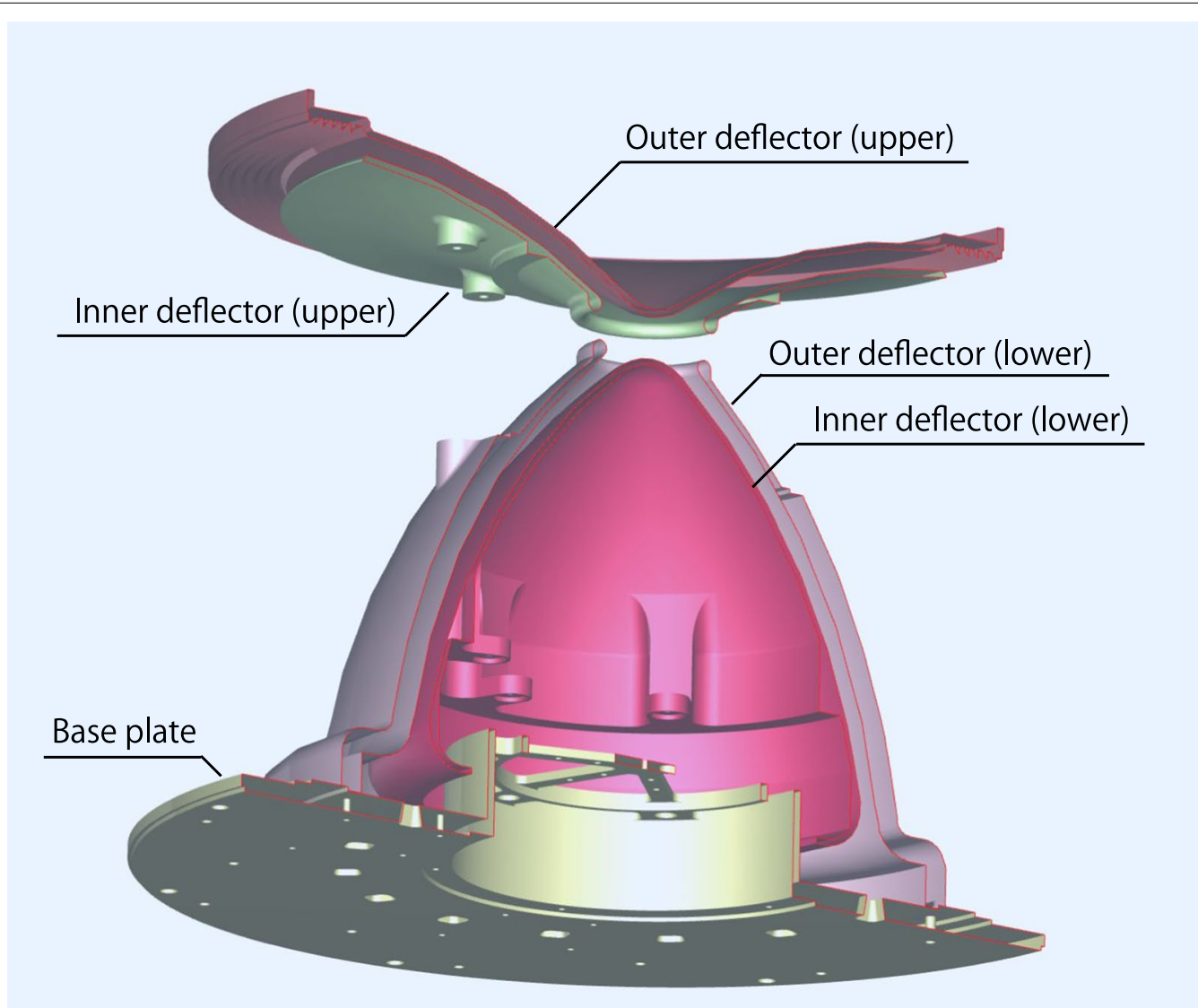

Fig. 5 Key structures of the cusp-type ESA (cross-sectional view). The outer deflectors (upper and lower), inner deflectors (upper and lower), and a base plate are displayed. High voltages are applied to the inner deflectors, while other structures are grounded. Detectors are mounted below the exit holes of the base plate 


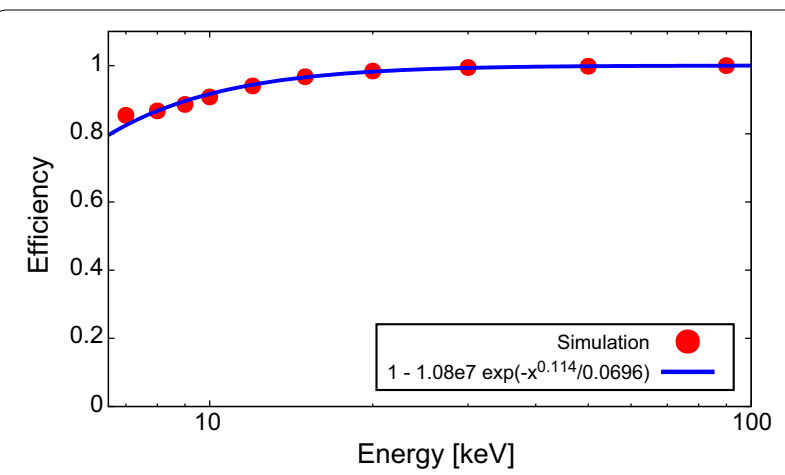

Fig. 6 APD efficiency curve based on the particle-in-matter simulation. The backscattering ratio $\beta(E)$ was obtained for varying incident energy $E$, and the efficiency $\varepsilon=1-\beta$ is plotted as a function of $E$ (red dots), with a fitted curve (blue)

energy dependence ensure a high reliability compared to the MCPs and CEMs, and these are the benefits of using the APD.

The APD also has the advantage over conventional solid-state detectors of measuring electrons below $<30 \mathrm{keV}$, because its internal gain provides a higher signal-to-noise ratio (Ogasawara et al. 2005, 2006, 2008, 2016; Kasahara et al. 2010, 2012). Figure 7 shows APDs mounted azimuthally on a board with resisters and capacitors. The effective area of an APD is $5 \mathrm{~mm} \times 5 \mathrm{~mm}$. Although a larger area was also considered in the design phase (Kasahara et al. 2010), the requirement for the minimum detectable energy $(<10 \mathrm{keV})$ suggested this detector size (note that the larger area causes an increased noise level, resulting in the higher minimum detectable energy). The thickness of the detectors is $50 \mu \mathrm{m}$, corresponding to a range of $\sim 75 \mathrm{keV}$ electrons (a fraction of $\sim 90 \mathrm{keV}$ electrons penetrate the detector, but they deposit sufficient energy for incident energy determination). Although the larger thickness enables better energy resolution at the higher energy $(>60 \mathrm{keV})$, the noise level deteriorates due to the larger bulk leakage current, since the noise due to the bulk leakage current dominates the capacitance noise (Kasahara et al. 2012). Furthermore, thicker detectors result in higher background count rates due to the $\gamma$-rays. On the other hand, a thinner detector (e.g., $<30 \mu \mathrm{m}$ ) leads to a significantly worse energy resolution or a critical underestimate of the incident energy at the higher energy, due to the penetration through the detector (Ogasawara et al. 2006). The final thickness is therefore the result of these trade-offs.

Note that the APD noise level is degraded by high temperature. In order to keep the minimum detectable energy below $10 \mathrm{keV}$, it is essential to achieve a low temperature. As shown in Fig. 2a, the sensor chassis is half covered by black kapton multilayered thermal insulators

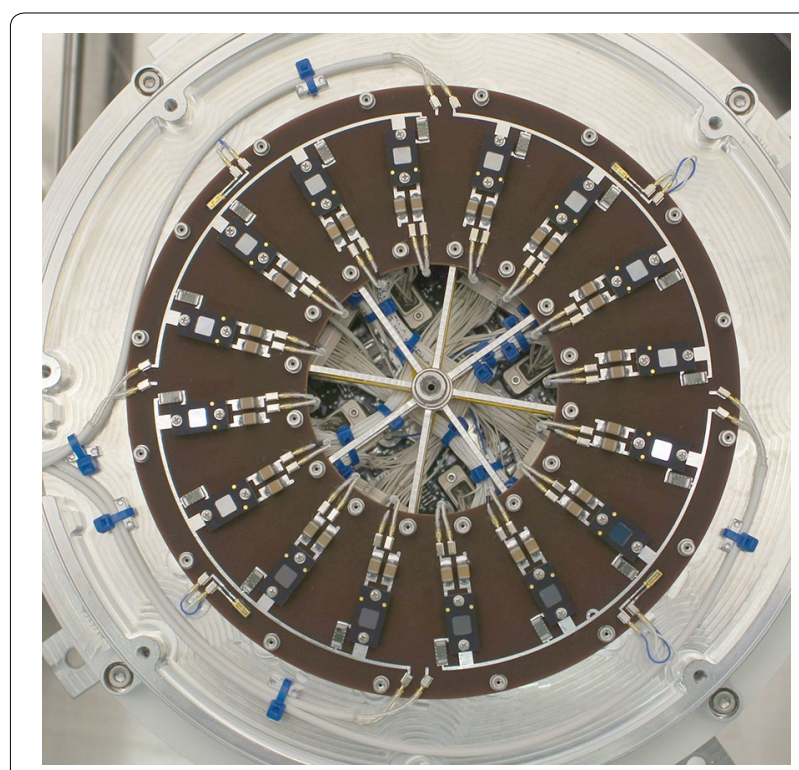

Fig. 7 Sixteen APDs mounted on a board. The high-voltage lines come from the left and are distributed in four sectors. There are four temperature sensors (AD590)

to avoid solar irradiation, while the other half side is painted white for radiative cooling against the dark sky (UPI WHITE LT48, made by UBE industries, Ltd.; it is conductive, and its solar absorptance and infrared emissivity are $\sim 0.2$ and $0.8-0.9$, respectively). Thus, the ESA works as a cooler for APDs. Furthermore, the APD board (shown in Fig. 7) and the ESA are thermally isolated from the rest of the instrument, which is a significant heat source due to the power consumption of the analog and digital electronics boards, by a thermally insulating polyimide structure. As a result, APDs are kept below $-10{ }^{\circ} \mathrm{C}$ throughout the orbit. There is no significant temperature difference among the 16 detectors (less than a few degrees centigrade).

\section{Energy coincidence method for background rejection}

Using both the ESA and APDs, two independent values indicating the electron incident energy can be compared. Such a two-parameter analysis enables effective background rejection, since independently measured energies are rarely the same for noise pulses, while they should be consistent for true signals (Kasahara et al. 2009).

The background rejection process is illustrated in Fig. 8. When a pulse is detected, the sensor FPGA checks whether the obtained $\mathrm{PH}$ is within a predetermined passband width $( \pm W)$ centered on the nominal value (PHO), for the particular azimuthal channel number and the ESA's SV (energy) step. In other words, for each detected signal, the FPGA process works as an energy band-pass filter for APDs. The width of the passband is set typically 


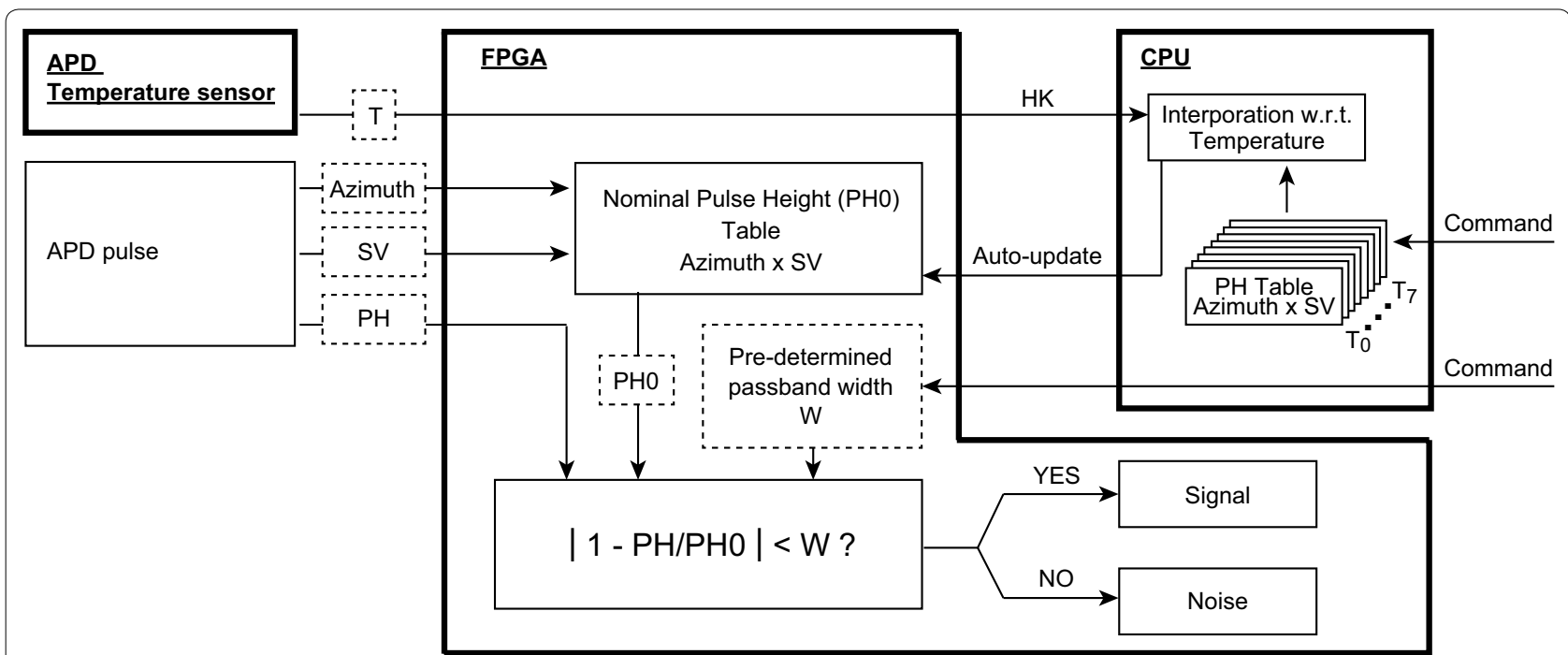

Fig. 8 Block diagram for the energy coincidence method. When an APD detects a pulse, its height (pulse height, PH) is compared with the nominal value (PHO) and determines whether it is signal or noise. The nominal value table is updated by the CPU software

at $\pm 14 \%$ (selectable by command) of the PHO. The table for PHO is constantly updated by the CPU software based on the measured temperature at the APDs. This is because the APD gain is temperature dependent (Kasahara et al. 2012), and thus, the PHO varies with temperature, but the FPGA does not have a sufficient memory capacity to hold multiple $\mathrm{PHO}$ tables for various temperature values.

The PH tables in the CPU software are written based on the in-flight data. For this purpose, the list data, from which the APDs' PH distribution can be produced, are acquired, as shown in "In-flight performance" section. Although the APD gain can also drift due to radiation damage (Kasahara et al. 2012), the long-term trends during the flight can be checked and the tables will be updated on the CPU accordingly.

Note that the passband does not fully cover the $\mathrm{PH}$ distributions of the true signals, as schematically shown in Fig. 9. Rather, some of the true signals (tails of the PH distribution) are discarded through this process, when a detected $\mathrm{PH}$ is outside the passband, even if it is a true signal. This results in an underestimate of the real flux. In order to compensate for this effect and obtain the corrected flux, the amounts of such discarded true signals are calculated. This is done by modeling the PH distribution with a Gaussian function (the asymmetry between the higher- and lower-energy sides are also taken into account using two Gaussian functions), which we found to be a good approximation for both the ground experimental data and in-flight data. The calculation of the error functions with the passband widths provides the fraction of the rejected true signals, and then, the noise-subtracted flux is multiplied by the compensation factor for this inadvertently rejected portion. This correction is done on the ground.

Figure 9 also illustrates that the background is not completely eliminated by this energy coincidence method, since the energy coincidence can occur by chance for the background pulses. Subtraction of these backgrounds on the ground may be important especially when the relativistic-energy electron flux is intense.

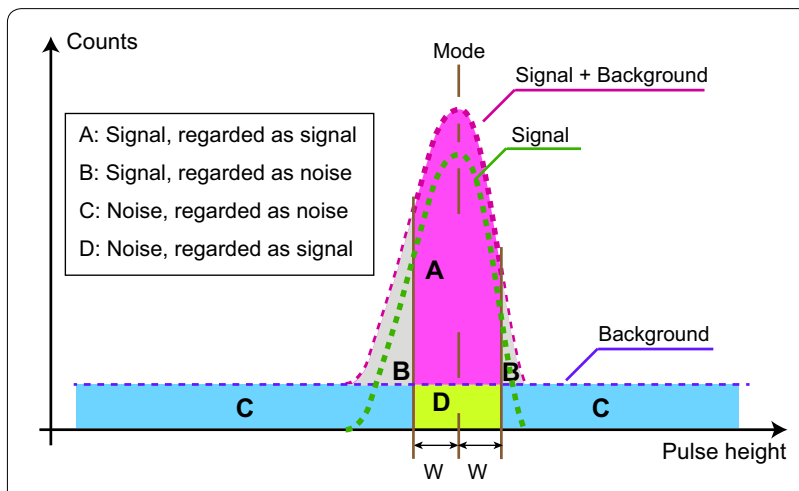

Fig. 9 Schematic showing the four categories in the PH distribution at energy coincidence checking. Inside the passband width indicated by $W$, the pulses are regarded as a true signal. However, a fraction of these come possibly from the background (category D), whereas most of them are from true signal (category A). The background pulses outside the passband (category $C$ ) are appropriately discarded as noise. The true signals outside the passband (category B) are inadvertently discarded as noise, and therefore, a correction is implemented. The correction factors were calculated using error functions, based on two half-Gaussian functions fitted separately for the higher and lower sides of the PH distribution 


\section{Evaluation of sensor specification}

The performances of the ESA and APDs are evaluated using a ground beam facility in Nagoya University, Japan. For the evaluation of the performance of the ESA, we utilized not only an electron but also a proton beam, since it is much easier to obtain straight and uniform proton beams, compared to electron beams, due to the geomagnetic field. Therefore, during a part of the calibration period, the ESA was connected to an external high-voltage-power supplier (HVPS) with an opposite polarity compared to the internal HVPS.

Figure $10 \mathrm{a}-\mathrm{c}$ represents the energy and angular responses of the ESA obtained with the proton beam. The contours in Fig. 10a indicate the relative transmittance. The laboratory result (magenta curves) agreed with the simulation (gray curves in Fig. 10a). The elevation indicates that the angle in a sensor's meridional plane and its origin is the horizontal direction $(Y-Z$ plane shown in Fig. 3a).
Figure 11a, b compares the energy and angle responses of the 16 azimuthal channels, respectively, indicating that the differences of the peak energies and angles were small compared to the energy resolution of all channels.

Figure 12a shows the azimuthal angular resolution. Each channel had a solution of $3.5^{\circ}$ FWHM. The gaps between the 16 channels are the dead areas. The blownup profile of a single channel is illustrated in Fig. $12 \mathrm{~b}$ and was well fitted by the simulation result.

After these verifications, we assembled the flight HV board and further checked the response to the electron beam. As we mentioned above, it is generally difficult to compare the simulation with the laboratory results for electron beams due to the deflection by the geomagnetic field. Nonetheless, we confirmed the predicted relativistic effect (e.g., at $70 \mathrm{keV}$, the SV value corresponding to the peak count shifted 7\% compared to the nonrelativistic case). Figure 10d shows the simulation result for $70 \mathrm{keV}$ with the relativistic effect (in gray), fitting well to the laboratory result for a $70 \mathrm{keV}$ electron beam (in cyan).

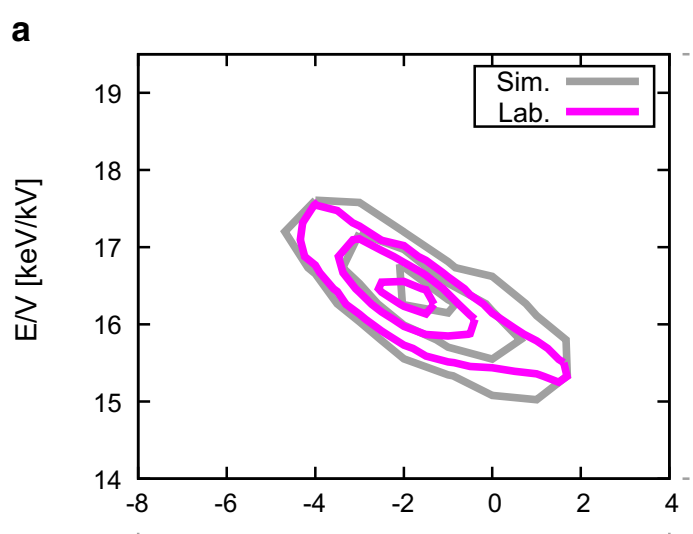

b

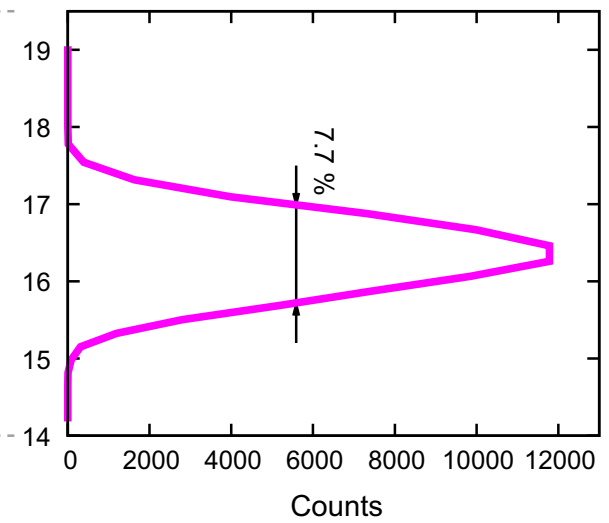

c

Fig. 10 Energy-angular response (azimuthal channel no. 0). a Contour lines of the transmittance for the energy and elevation angle (10, 50, and $90 \%$ of the peak are outlined). The results of the proton beam experiment and simulation are represented in magenta and gray, respectively. $\mathbf{b}$ Response curve for the elevation angle, $\mathbf{c}$ response for the incident energy, and $\mathbf{d}$ transmission contour for the relativistic case (the electron beam results and relativistic calculation are shown in cyan and gray, respectively) 

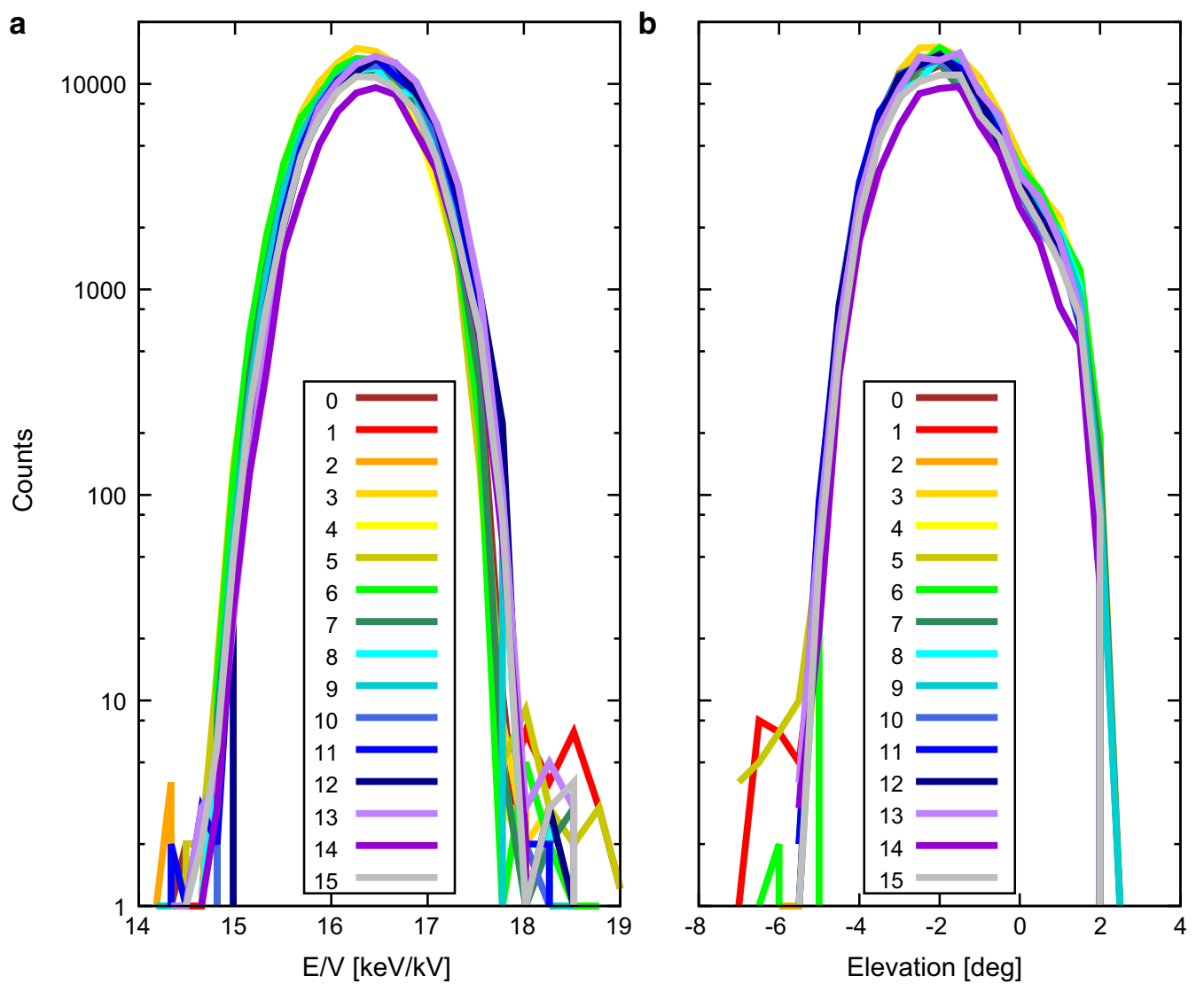

Fig. 11 Energy and angular responses of the 16 channels for the fixed proton energy of $40 \mathrm{keV}$. For the energy profiles, the a elevation angle dependence was integrated from $-5.5^{\circ}$ to $2.5^{\circ}$, and $\mathbf{b}$ for elevation angle profile, the SV dependence was integrated from 2.10 to $2.82 \mathrm{kV}$

Through these results, we confirmed that the analyzer was manufactured and assembled properly as per the design. In addition, we checked the EUV rejection, using a $\mathrm{D}_{2}$ lamp with a photon flux similar to the solar irradiance. We confirmed there was no increase in noise from the background level.

The response of APDs was also tested with an electron beam. The pulse height peaks of the output signals were in a quasi-linear relation with the incident electron energy, as shown in Fig. 13 with the black circles indicating the peaks of the pulse height distributions at several incident energies and the gray shading representing the full width at half maximums of the same distributions. The data were obtained for $27-28{ }^{\circ} \mathrm{C}$, much higher than in orbit, as this test was conducted in the vacuum chamber without active control of the temperature, and therefore, there was substantial thermal emission from the wall of the vacuum chamber.

\section{Operation mode and data product}

Here we introduce the science/engineering data products of the MEP-e. When the MEP-e is in the normal mode, it produces count and list data. In the table-dump mode, the MEP-e sends the table data for a read-back check. The structures of each data set are described below. The CPU software receives data from the sensor FPGA and then transmits the data to the mission data processor after compression.

\section{Normal mode}

The MEP-e produces a packet of $1.756 \mathrm{kB}$ per one energy sweep ( $=1 / 32$-spin). The packet consists of two types of science data blocks, i.e., "count data" and "list data." The whole structure is shown in Table 2.

\section{Count data}

The sensor FPGA prepares two 12-bit counters for each SV step. One is for the energy coincidence counts (including only signals for which the two energy determinations are consistent), and the other is for all counts (the result of the energy check result is not considered). For the purpose of the ground check, both types of count data are dumped. Thus, there are $2 \times 12$ bit $\times 16 \mathrm{SV}$ 

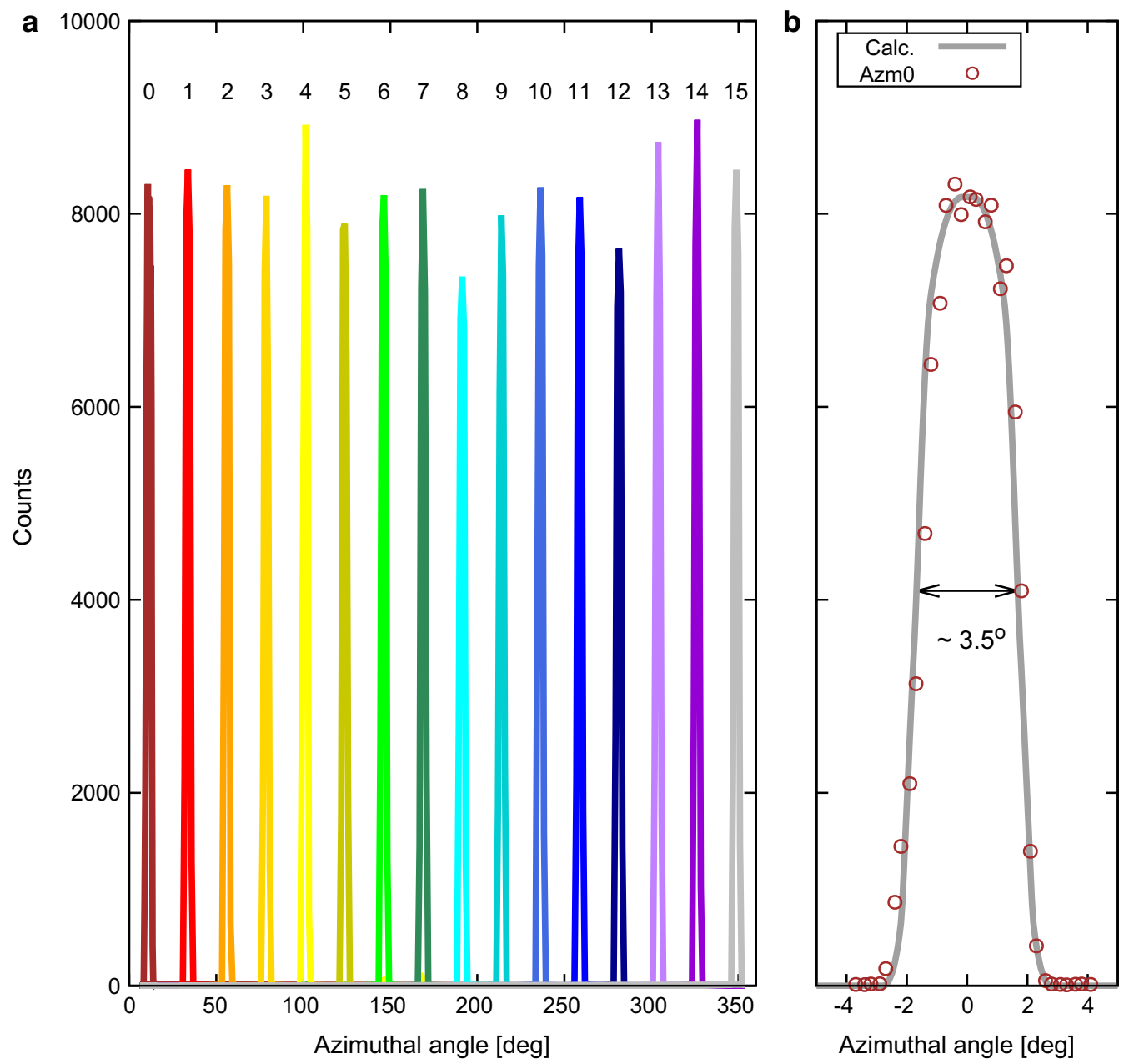

Fig. 12 Azimuthal angular responses of $\mathbf{a} 16$ channels and $\mathbf{b}$ APD channel 0 . The latter was blown up and compared to the simulation result (shown as the gray curve)

steps $\times 16$ azimuthal channels $=768$ bytes for the count data per energy sweep.

\section{List data}

In addition to the count data, list data are also produced. This data type contains the information of the APD's pulse height as well as the ESA energy step for each incoming particle and is mainly used for the in-flight calibration. The size of a data packet is 6 bytes per event (see Table 2 for the contents of the packet). Considering the expected maximum count rate is 5000 counts/s for one azimuthal channel, it is not reasonable to produce list data for all events (in that case, the data product rate is $7.5 \mathrm{kB}$ per SV step, far beyond the capacity of the system data recorder and the down link rate). For this reason, the production of the list data packets is restricted to 10 events per SV step on a first-come basis (then the size of the list data is 960 bytes per spin phase).

\section{Table-dump mode}

In this mode, the sensor tables for the SV, threshold, and energy coincidence are dumped instead of the count data. No scientific data are acquired in this mode. This mode is mainly used for the check of the written table data.

\section{Compression}

The data size in Table 1 is for raw data (i.e., before compression). The software applies lossless compression (a few types of algorithms were prepared) before the data dump. When further compression is required to keep the system data recorder from filling up, we need to reduce the data by several other methods. In such a case, one of the reduction modes and degree of reduction in Table 3 


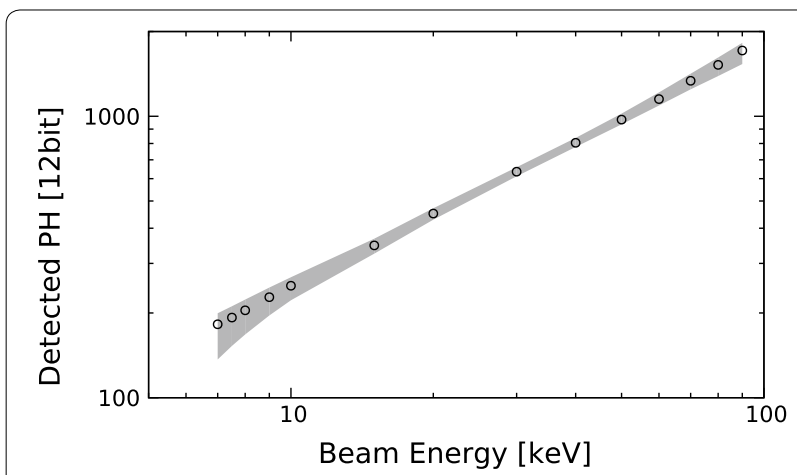

Fig. 13 Relationship between the electron incident energies and signal pulse heights obtained in the laboratory. The circles indicate the peaks of the $\mathrm{PH}$ distributions at corresponding energies for $27-28^{\circ} \mathrm{C}$. The applied HV to the APD was approximately $-180 \mathrm{~V}$. The width of the gray shaded band illustrates the $1 \sigma$ range of the $\mathrm{PH}$ distribution varying with the incident energy. The standard deviation $\sigma$ is not the same for the upper and lower sides of the peak, as they are derived from fittings with two different half-Gaussians for the upper and lower halves of the PH distributions. In order to obtain this data set, the sensor was irradiated with electron beams of varying incident energies ranging within 7-90 keV in the laboratory

Table 2 Normal data of the MEP-e

\begin{tabular}{|c|c|c|}
\hline Size (bytes) & Data & Notes \\
\hline 28 & Header & $\begin{array}{l}\text { Time indicator, spin phase, } \\
\text { etc }\end{array}$ \\
\hline 384 & $\begin{array}{l}\text { 12-bit counter: } \\
\text { SV(16) × Azm (16) }\end{array}$ & Noise-reduced data \\
\hline 384 & $\begin{array}{l}\text { 12-bit counter: } \\
\text { SV(16) × Azm (16) }\end{array}$ & Raw (noise-remaining) data \\
\hline 960 & $\begin{array}{l}\text { List data ( } 6 \text { bytes per event, } \\
160 \text { events at maximum, } \\
\text { first-come base) }\end{array}$ & $\begin{array}{l}\text { One event includes SV step } \\
\text { ( } 4 \text { bits), pulse height ( } 12 \\
\text { bits), azimuth channel ( } 4 \\
\text { bits), APD temperature ( } 6 \\
\text { bit } \times 4 \text { ), and SV table ID ( } 1 \\
\text { bit). } 10 \text { events per SV step } \\
\text { at maximum }\end{array}$ \\
\hline
\end{tabular}

A packet is produced for every spin phase

Table 3 Reduction mode list

\begin{tabular}{|c|c|}
\hline Mode & Notes \\
\hline Raw & No reduction is implemented \\
\hline Superposition & $\begin{array}{l}n \text {-spin accumulated. } n=2^{i}(i=1,2,3,4), i \text { can be } \\
\text { selected by command }\end{array}$ \\
\hline Snapshot & $\begin{array}{l}\text { Non-reduced full spin data are obtained once per } n \\
\text { spins. }(n-1) / n \text { data are discarded. } n=2^{i}(i=1,2,3,4) \text {, } \\
i \text { can be selected by command }\end{array}$ \\
\hline Spin-phase skip & $\begin{array}{l}\text { Depending on the degree } i \text {, data in below spin phases } \\
\text { are obtained, while others are discarded. } \\
(i=1) 0 / 2 / 4 / 6 / 8 / 10 / 12 / 14 / 16 / 18 / 20 / 22 / 24 / 26 / 28 / 30 \\
(i=2) 0 / 4 / 8 / 12 / 16 / 20 / 24 / 28 \\
(i=3) 0 / 4 / 8 / 12 \\
(i=4) 0 / 8 \\
\text { The degree } i \text { can be selected by command }\end{array}$ \\
\hline
\end{tabular}

is selected. These reduction modes are basically set by timeline commands.

\section{S-WPIA data}

One of the key objectives of the MEP-e (and ERG) is to quantify the energy transfer between particles and electromagnetic waves (Katoh et al. 2013; Hikishima et al. 2014). Of special interest is the Landau/gyro-resonance between electrons and whistler chorus waves. Although previous observations have addressed this issue, their focus has been limited to the correlation between the electron flux intensity and/or pitch-angle distribution and wave intensification. The critical problem of this approach is that not possible to distinguish cause and effect, or, in other words, the direction of the energy transfer. In order to unambiguously verify the wave growth or particle energization, it is essential to determine the particle velocity vector with the time resolution that is short enough compared to the period of whistler chorus waves. This requires a time resolution of tens of microseconds or better. However, if the count data packets are regularly produced in this time resolution, the data size and rate easily exceed the capacity of the system data recorder and the down link rate. In addition, precise synchronization is required between the particle and wave instruments with a time resolution of the order of microseconds.

In order to challenge this issue, a software-type waveparticle interaction analysis (S-WPIA) was implemented onboard the ERG. In this framework, three electron sensors, MEP-e, HEP, and XEP (Higashio et al. 2017), are directly connected to the wave instrument PWE (see Fig. 4). In order to synchronize the particle data with the wave data, an "S-WPIA clock" of $524.288 \mathrm{kHz}$ is distributed from the PWE to the electron sensors. These electron sensors send an "S-WPIA event packet" for each event (i.e., particle detection) to the mission data recorder (MDR), when the "S-WPIA generation flag" is ON. This flag is distributed via a shared data packet, circulating in the mission system network. The CPU application software checks the flag once per second. The PWE also sends the wave data to the MDR, and the S-WPIA application calculates the physical values related to wave growth and particle energization. In addition to the calculated values, the raw burst data from the MEP-e, HEP, XEP, and PWE of short durations are also dumped.

Table 4 shows the S-WPIA packets of the MEP-e. The event packet includes the S-WPIA clock and information on each incoming particle (the directions are determined by the spacecraft spin phase and azimuthal channel and the energy). We emphasize that this S-WPIA clock is crucial for unprecedented high time resolution. This clock ensures that the MEP-e and PWE are synchronized 
Table 4 S-WPIA data of MEP-e

\begin{tabular}{lcl}
\hline Packet type & Size (bytes) & Data \\
\hline Tl packet & 6 & Tl data for clock synchronization \\
Counter packet & 12 & Event counter (used for data checking) \\
Event packet & 6 & $\begin{array}{l}\text { S-WPIA clock (24 bits), spin phase (5 bits), } \\
\text { SV step (4 bits), energy coincidence } \\
\text { flag (1 bit), azimuth channel (4 bits), SV } \\
\text { table ID (1 bit) }\end{array}$ \\
& & Dummy data for tests \\
\hline
\end{tabular}

in a time resolution of $\sim 2 \mu \mathrm{s}$, enabling analyses of the energy transfer between the wave and particle. This high time resolution is not for absolute time but for the relative timing between the MEP-e and PWE. For the waveparticle interaction analyses, only the latter is an issue. Since the S-WPIA clock is reset at the spin pulse, the TI packet is also needed for synchronization, determining the coarse time information. Counter and dummy packets are used to check the data quality and function. More details are described in other papers regarding this issue (Katoh et al. 2018).

\section{In-flight performance}

After more than 1 month of hibernation of the particle instruments during the initial spacecraft critical phases, the MEP-e was first turned-on on January 30, 2017. The
Table 5 Energy steps of the ESA measurements

\begin{tabular}{lll}
\hline Step no. & Central energy (keV) & Applied voltage (V) \\
\hline 0 & $7.0-87.5$, increasing & $427-4958$ \\
1 & 87.5 & 4958 \\
2 & 72.7 & 4164 \\
3 & 60.4 & 3499 \\
4 & 50.3 & 2939 \\
5 & 42.0 & 2468 \\
6 & 35.0 & 2072 \\
7 & 29.3 & 1740 \\
8 & 24.5 & 1461 \\
9 & 20.5 & 1226 \\
10 & 17.1 & 1029 \\
11 & 14.3 & 864 \\
12 & 12.0 & 725 \\
13 & 10.1 & 608 \\
14 & 8.4 & 510 \\
15 & 7.0 & 427 \\
\hline
\end{tabular}

HVPS initial turn-on was conducted after the other particle instruments. The nominal voltages were successfully applied without any sign of discharges. These initial checkouts were by real-time commands. The routine operations by timeline commands started on March 23, 2017.

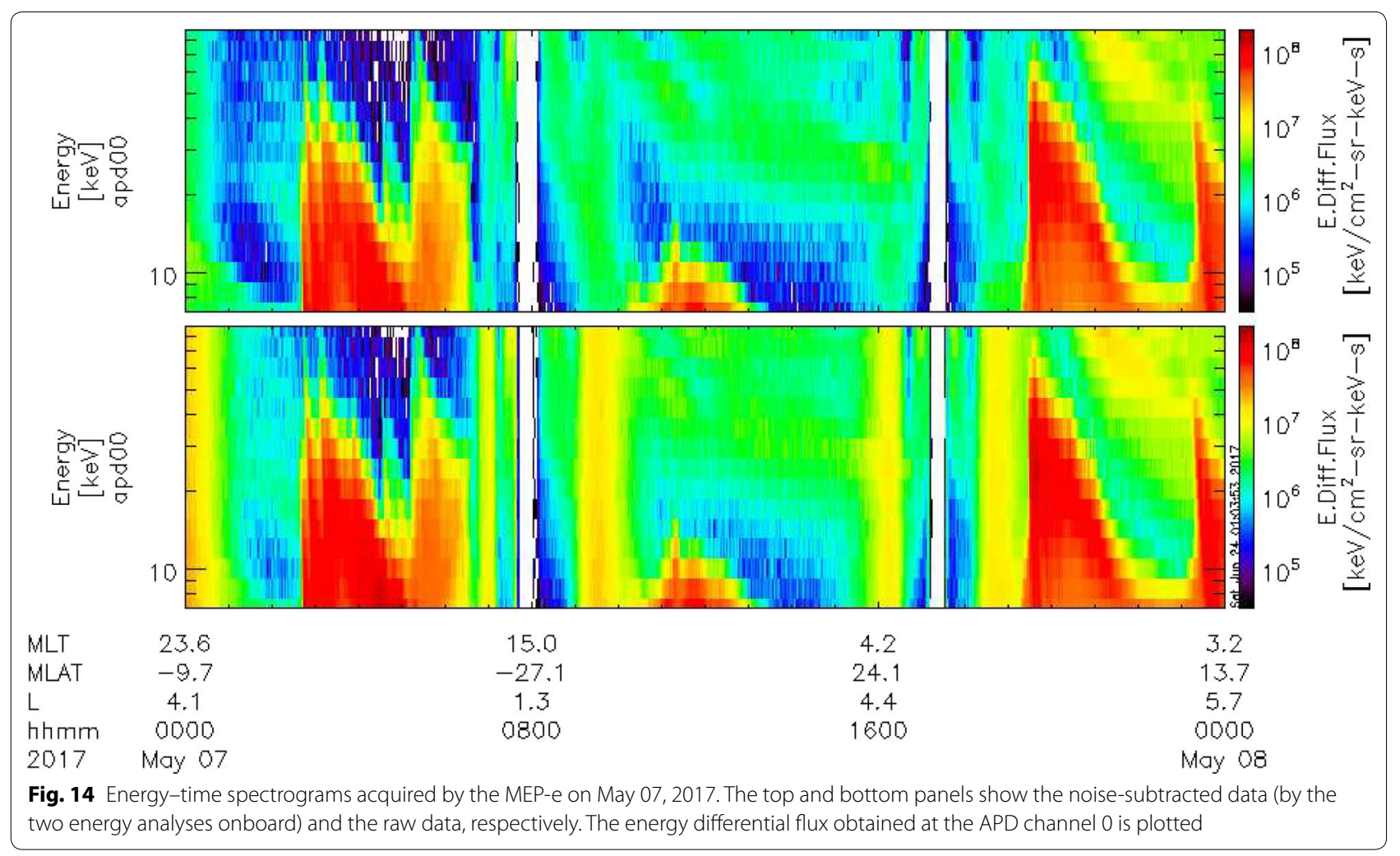


Figure 14 shows the 24-h-long energy-time spectrograms on May 07, 2017. Since the orbiting period was $\sim 9.5 \mathrm{~h}$, this plot covers more than two revolutions. Perigee passes are seen as white blanks at 8:00 and 17:30 UT. This is partly because the $\mathrm{HV}$ is turned off during perigee passes to avoid significant damages due to penetrating protons $(>50 \mathrm{MeV})$, and therefore, data are not obtained. Around the perigee passes $(L$ value $<3)$, the sensor is in a "2-spin superposition" mode (cf., Table 3), in order to reduce the data rate. The top panel illustrates the energychecked (i.e., background-subtracted) data, whereas the bottom panel shows the raw data. The effectiveness of the background subtraction is clear at the outer radiation belt region (at around 0:00-1:00 UT, 7:00 UT, 9:00-10:00 UT, 16:00-16:30 UT, 18:30-19:00 UT), as well as at the inner radiation belt (both sides of the white blanks near perigee). The pronounced flux variabilities, including dispersion-less and dispersive injections, occurred frequently throughout the MEP-e's measurement energy range.

The nominal energy steps of the ESA and corresponding voltages are shown in Table 5 as an example. The applied SV value changes logarithmically. Note that the energy is not precisely proportional to the voltage due to the relativistic effect. In nominal operations, care should

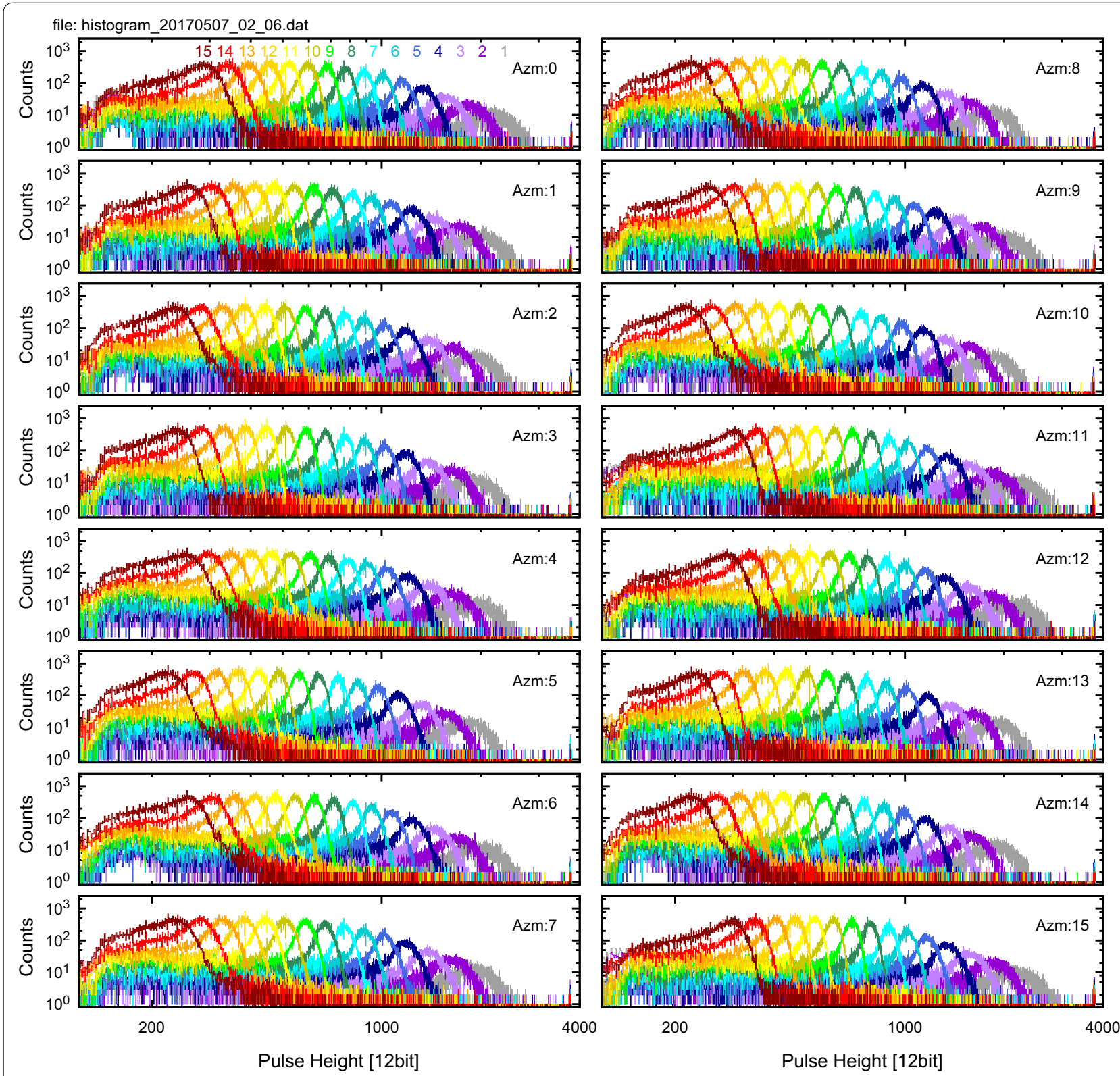

Fig. 15 Pulse height histograms for 16 channels. The colors of lines indicate the energy steps. These were reproduced from list data accumulated for 2-6 UT on May 07, 2017 (the same day as Fig. 14) 


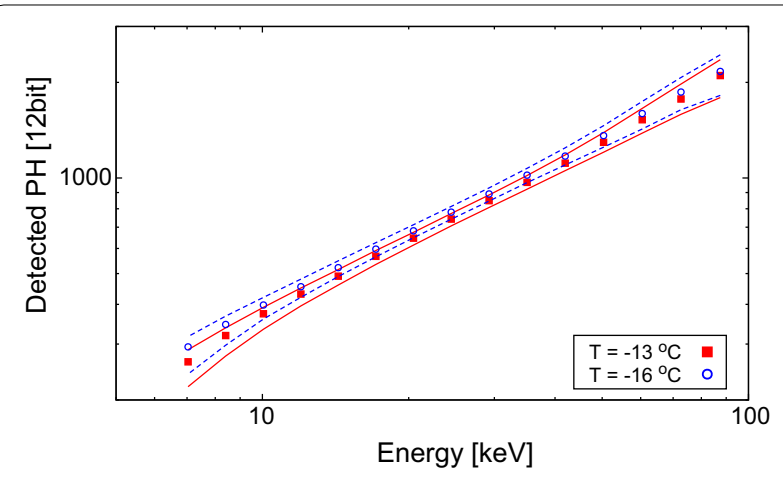

Fig. 16 Relationship between the ESA measurement energy and signal pulse height obtained from the in-flight data. The red filled squares indicate the peaks of the PH distributions at corresponding energy steps of $-13^{\circ} \mathrm{C}$, while the width of the red band illustrates the $1 \sigma$ range of the distribution at the same temperature, similar to Fig. 13. The blue circles and dashed bands are those at $-16^{\circ} \mathrm{C}$. The temperature dependence of the gain is $\sim-1.5 \% / K$, consistent with previous laboratory experiments (Kasahara et al. 2012)

be taken in using the data in the "Oth" SV step. The SV rise time for full output ( $5 \mathrm{kV}$ ) takes $>5 \mathrm{~ms}$ (as Fig. $3 \mathrm{c}$ schematically illustrates), and thus, electrons with a variety of energies can be detected in this step. The data in the 0th step are thus not useful for scientific data analyses for this nominal SV table operation. In addition, the flight data (as well as laboratory calibration data) showed some noise counts when the SV increases up to $5 \mathrm{kV}$ in the 0th step (only in the azimuth channel 11, closest to the cable distributing SV to the lower-inner deflector of the ESA). On the other hand, no noise (due to SV stepping) is recognized in other SV steps. Also, no apparent signature of the EUV photon background is seen in any channel, as expected from the laboratory tests.

Pulse height distributions of 16 APDs are shown in Fig. 15. These histograms were reproduced from the list data on the same day as shown in Fig. 14 ( $4 \mathrm{~h}$ from 2 to 6 UT). The colors correspond to the energy step defined by the ESA. The peak of the lowest energy $(7 \mathrm{keV})$ is well above the electronics threshold ( $\mathrm{PH} \sim 150 \mathrm{bit})$. The $\mathrm{PH}$ profiles shown in Fig. 15 are used for the calibration of the background-subtracted flux data (described in "Energy coincidence method for background rejection" section). A monotonic relationship between the energy and $\mathrm{PH}$ is common for all APDs. As an example, the energy dependency of the peak and width of the pulse height distributions of the azimuth channel 0 are shown in Fig. 16 for two temperature conditions. The gain dependence on temperature $(\sim-1.5 \% / \mathrm{K})$ is consistent with ground laboratory experiments (Kasahara et al. 2012). The temperature variation in orbit is only $\sim 3{ }^{\circ} \mathrm{C}$, as a result of the successful temperature design and heater control system.

\section{Summary}

The MEP-e was developed for providing mediumenergy electron measurements by ERG, and observations have now begun. It detects electrons with energies of 7-87 keV and obtains velocity distribution functions, which are the key to understanding the formation and decay of the radiation belt. Observations in combination with other instruments onboard the ERG, other magnetospheric explorers (Angelopoulos et al. 2008; Burch et al. 2016; Escoubet et al. 1997; Mauk et al. 2013; Nishida et al. 1994), as well as ground-based observatories (e.g., Shiokawa et al. 2017) and modeling output (Seki et al. 2018) are expected to shed new light on radiation belt physics.

\section{Authors' contributions}

SK designed, assembled, and tested the MEP-e hardware, as well as coded the application software. SY contributed in all aspects of the engineering/flight model development, including the assembly, environment tests, and ground calibration. TM, KA, TT, and MH supported the development of the MEP-e since the breadboard model phase. MH also developed the calibration facility. YS supported the thermal design of the MEP-e. All authors read and approved the final manuscript.

\section{Author details}

${ }^{1}$ Department of Earth and Planetary Science, School of Science, The University of Tokyo, 7-3-1, Hongo, Bunkyo-ku, Tokyo, Japan. ${ }^{2}$ Osaka University, Machikaneyama-cho, Toyonaka, Japan. ${ }^{3}$ Institute of Space and Astronautical Science, 3-1-1, Yoshinodai, Chuo-ku, Sagamihara, Kanagawa, Japan. ${ }^{4}$ Institute for Space-Earth Environmental Research, Nagoya University, Furo-cho,

Chikusa-ku, Nagoya, Aichi 24105, Japan.

\section{Acknowledgements}

Development of the MEP-e was accomplished by the remarkable efforts of engineers in Mitsubishi Heavy Industries, Ltd., Meisei Electric Co., Ltd., and YS Design Co., Ltd. K. Mori and Minodenshi Co., Ltd., are also recognized for the design and fabrication of the amplifier board, which is the core of this instrument. We thank the reviewers of the instrument design reviews, $T$. Mukai, Y. Saito, S. Watanabe, I. Yoshikawa, and T. Kii, for their useful and critical comments. We are also grateful to L. Kistler for her assistance in improving this paper during her stay in the University of Tokyo. This work was partly supported by JSPS Kakenhi Grant No. 07J01222. The outstandingly dedicated works of the ERG Project members (and support from their families) were indispensable for the MEP-e and ERG spacecraft.

\section{Competing interests}

The authors declare that they have no competing interests.

Ethics approval and consent to participate

Not applicable.

\section{Publisher's Note}

Springer Nature remains neutral with regard to jurisdictional claims in published maps and institutional affiliations.

Received: 4 July 2017 Accepted: 19 April 2018

Published online: 02 May 2018 


\section{References}

Angelopoulos V (2008) The THEMIS mission. Space Sci Rev 141(1):5. https://doi. org/10.1007/s11214-008-9336-1

Asamura K, et al (2017) Observations of low-energy ions with Arase/LEPi, In: American Geophysical Union fall meeting

Burch JL, Moore TE, Torbert RB, Giles BL (2016) Magnetospheric multiscale overview and science objectives. Space Sci Rev 199(1):5-21. https://doi. org/10.1007/s11214-015-0164-9

Carlson CW, McFadden JP (2013) Design and application of imaging plasma instruments. In: Pfaff RF, Borovsky JE, Young DT (eds) Measurement techniques in space plasmas: particles. American Geophysical Union, Washington DC, pp 125-140. https://doi.org/10.1029/GM102p0125

Escoubet CP, Schmidt R, Goldstein ML (1997) Cluster - science and mission overview. Space Sci Rev 79(1):11-32. https://doi.org/10.102 3/A:1004923124586

Funsten HO, Skoug RM, Guthrie AA, MacDonald EA, Baldonado JR, Harper RW, Henderson KC, Kihara KH, Lake JE, Larsen BA, Puckett AD, Vigil VJ, Friedel RH, Henderson MG, Niehof JT, Reeves GD, Thomsen MF, Hanley JJ, George DE, Jahn J-M, Cortinas S, De Los Santos A, Dunn G, Edlund E, Ferris M, Freeman M, Maple M, Nunez C, Taylor T, Toczynski W, Urdiales C, Spence HE, Cravens JA, Suther LL, Chen J (2013) Helium, oxygen, proton, and electron (HOPE) mass spectrometer for the radiation belt storm probes mission. Space Sci Rev 179:423-484

Higashio N, et al (2017) Energy dependence of relativistic electron variations in the outer radiation belt during the recovery phase of magnetic storms: Arase/XEP observation. In: American Geophysical Union fall meeting

Hikishima M, Omura Y, Summers D (2010) Self-consistent particle simulation of whistler mode triggered emissions. J Geophys Res Space Phys 115(A12):A12246. https://doi.org/10.1029/2010JA015860

Hikishima M, Katoh Y, Kojima H (2014) Evaluation of waveform data processing in wave-particle interaction analyzer. Earth Planets Space 66(1):63. https://doi.org/10.1186/1880-5981-66-63

Horne RB, Thorne RM, Shprits YY, Meredith NP, Glauert SA, Smith AJ, Kanekal SG, Baker DN, Engebretson MJ, Posch JL, Spasojevic M, Inan US, Pickett JS, Decreau PME (2005) Wave acceleration of electrons in the Van Allen radiation belts. Nature 437:227-230

Horne RB, Thorne RM, Glauert SA, Meredith NP, Pokhotelov D (2007) Santol'ık, O.: Electron acceleration in the Van Allen radiation belts by fast magnetosonic waves. Geophys Res Lett 34(17):L17107. https://doi. org/10.1029/2007GL030267

Joy DC (1991) An introduction to Monte Carlo simulations. Scanning MicrosC 5:329-337

Kasahara S, Asamura K, Saito Y, Takashima T, Hirahara M, Mukai T (2006) Cusp type electrostatic analyser for measurements of medium energy charged particles. Rev Sci Instrum 77:123303

Kasahara S, Asamura K, Ogasawara K, Kazama Y, Takashima T, Hirahara M, Saito $Y$ (2009) A noise attenuation method for the medium-energy electron measurements in the radiation belt. Adv Space Res 43(5):792-801

Kasahara S, Takashima T, Asamura K, Mitani T (2010) Development of an APD with large area and thick depletion layer for energetic electron measurements in space. IEEE Trans Nucl Sci 57(3):841-847

Kasahara S, Takashima T, Hirahara M (2012) Variability of the minimum detectable energy of an APD as an electron detector. Nuclear Inst Methods Phys Res A 664(1):282-288

Kasahara Y et al (2017) The plasma wave experiment (PWE) on board the Arase (ERG) satellite initial results and collaboration with the ground network stations and Van Allen Probes. In: American Geophysical Union fall meeting

Katoh Y, Omura Y (2006) A study of generation mechanism of VLF triggered emission by self-consistent particle code. J Geophys Res 111:12207

Katoh Y, Omura Y (2007) Relativistic particle acceleration in the process of whistler-mode chorus wave generation. Geophys Res Lett 34(13):L13102. https://doi.org/10.1029/2007GL029758

Katoh Y, Kitahara M, Kojima H, Omura Y, Kasahara S, Hirahara M, Miyoshi Y, Seki K, Asamura K, Takashima T, Ono T (2013) Significance of wave-particle interaction analyzer for direct measurements of nonlinear wave-particle interactions. Ann Geophys 31(3):503-512. https://doi.org/10.5194/ angeo-31-503-2013

Katoh Y et al (2018) Software-type Wave-Particle Interaction Analyzer on board the Arase satellite. Earth Planets Space 70:4. https://doi.org/10.1186/ s40623-017-0771-7
Kennel CF, Petchek HE (1966) Limit on stably trapped particle fluxes. J Geophys Res 71:1-28

Mauk BH, Fox NJ, Kanekal SG, Kessel RL, Sibeck DG, Ukhorskiy A (2013) Science objectives and rationale for the radiation belt storm probes mission. Space Sci Rev 179(1):3-27. https://doi.org/10.1007/s11214-012-9908-y

Mitani T, et al (2017) "High energy Electron exPeriment (HEP)" onboard the ERG satellite, American Geophysical Union fall meeting

Miyoshi Y, et al (2017) Arase: mission overview and initial results. In: American Geophysical Union fall meeting

Nishida A (1994) The geotail mission. Geophys Res Lett 21(25):2871-2873. https://doi.org/10.1029/94GL01223

Ogasawara K, Asamura K, Mukai T, Saito Y (2005) Avalanche photodiode for measurement of low-energy electrons. Nucl Instrum Methods Phys Res Sect A 545(3):744-752. https://doi.org/10.1016/j.nima.2005.02.026

Ogasawara K, Takashima T, Asamura K, Saito Y, Mukai T (2006) The effect of depletion layer thickness in avalanche photodiodes for measurement of low-energy electrons. Nucl Instrum Methods Phys Res Sect A 566(2):575583. https://doi.org/10.1016/j.nima.2006.08.001

Ogasawara K, Hirahara M, Miyake W, Kasahara S, Takashima T, Asamura K, Saito Y, Mukai T (2008) High-resolution detection of 100 keV electrons using avalanche photodiodes. Nucl Instrum Methods Phys Res Sect A 594(1):50-55. https://doi.org/10.1016/j.nima.2008.05.056

Ogasawara K, Livi SA, Allegrini F, Broiles TW, Dayeh MA, Desai MI, Ebert RW, Llera K, Vines SK, McComas DJ (2016) Next-generation solid-state detectors for charged particle spectroscopy. J Geophys Res Space Phys 121(7):6075-6091. https://doi.org/10.1002/2016JA022559.2016JA022559

Omura Y, Summers D (2006) Dynamics of high-energy electrons interacting with whistler mode chorus emissions in the magnetosphere. J Geophys Res Space Phys 111(A9):A09222. https://doi.org/10.1029/2006JA011600

Omura Y, Katoh Y, Summers D (2008) Theory and simulation of the generation of whistler-mode chorus. J Geophys Res Space Phys 113(A4):A04223. https://doi.org/10.1029/2007JA012622

Seki K, Miyoshi Y, Ebihara Y, Katoh Y, Amano T, Saito S, Shoji M, Nakamizo A, Keika K, Hori T, Nakano S, Kamiya K, Takahashi N, Omura Y, Nose M, Fok M-C, Tanaka T, leda A, Yoshikawa A (2018) Theory, modeling and integrated studies in the Arase (ERG) project. Earth Planets Space 70:17. https://doi.org/10.1186/s40623-018-0785-9

Shiokawa K et al (2017) Ground-based instruments of the PWING project to investigate dynamics of the inner magnetosphere at subauroral latitudes as a part of the ERG-ground coordinated observation network. Earth Planets Space 69:160. https://doi.org/10.1186/s40623-017-0745-9

Summers D, Thorne RM, Xiao F (1998) Relativistic theory of wave-particle resonant diffusion with application to electron acceleration in the magnetosphere. J Geophys Res Space Phys 103(A9):20487-20500. https://doi. org/10.1029/98JA01740

\section{Submit your manuscript to a SpringerOpen ${ }^{\circ}$ journal and benefit from:}

- Convenient online submission

- Rigorous peer review

- Open access: articles freely available online

- High visibility within the field

- Retaining the copyright to your article

Submit your next manuscript at springeropen.com 